\title{
Challenging Treatment of a Female Patient with Extensive Fournier's Gangrene - Case Report
}

Ognen Kostovski ${ }^{1}$, Olivera Spasovska', Gjorgji Trajkovski', Svetozar Antovic ${ }^{1}$, Irena Kostovska ${ }^{2}$, Katerina Tosheska-Trajkovska ${ }^{2}$, Biljana Kuzmanovska ${ }^{3}$, Sofija Pejkova ${ }^{4}$, Nikola Jankulovski ${ }^{1}$

${ }^{1}$ University Clinic of Abdominal Surgery, Faculty of Medicine, Ss. Cyril and Methodius University in Skopje, Skopje, North Macedonia;

${ }^{2}$ Department of Medical Biochemistry, Faculty of Medicine, Ss. Cyril and Methodius University in Skopje, Skopje, North Macedonia;

${ }^{3}$ University Clinic of Traumatology, Orthopedic Disease, Anesthesiology, Reanimation and Intensive Care Medicine and Emergency Department, Faculty of Medicine, Ss. Cyril and Methodius University in Skopje, Skopje, North Macedonia; ${ }^{4}$ University Clinic of Plastic and Reconstructive Surgery, Faculty of Medicine, Ss. Cyril and Methodius University in Skopje, Skopje, North Macedonia Received June 16, 2020; Accepted January 28, 2021.

Key words: Fournier's gangrene - Debridement - Negative pressure wound therapy

Abstract: Fournier's gangrene $(F G)$ is a necrotizing fasciitis of the genital, perianal and perineal regions, caused by multiple anaerobic/aerobic infection. It is a rare but very serious condition with multiple long-term complications and high mortality rate. Early diagnosis and multidisciplinary approach in treatment of complicated cases of FG are crucial to the successful outcome. We report a case of an extensive FG in a 59-years-old female patient with multiple risk factors such as obesity, type 2 diabetes and hypertension. She was hospitalized as an emergency case with diabetic ketoacidosis, sepsis and extensive necrotic lesions located perineal, perianal, genital and spread to inguinal, hypogastric, gluteal and sacrococcygeal region. Fournier's gangrene was diagnosed, and after prompt resuscitation, intravenous fluids, broad-spectrum antibiotics, insulin infusion, emergency aggressive surgical debridement was performed. Several aerobic and anaerobic bacteria were isolated from wound culture and hemoculture. Patient has second debridement after four

Mailing Address: Irena Kostovska, MD., PhD., Department of Medical Biochemistry, Faculty of Medicine, Ss. Cyril and Methodius University, Skopje, North Macedonia, 50 Divizija 6, 1000 Skopje, North Macedonia; Phone: +389 782089 90; e-mail: irenakostovska22@yahoo.com 
days. After second debridement was applied metabolic control, broad-spectrum antibiotics coverage, dressing the wound and negative pressure wound therapy (NPWT). Patient was discharged home five weeks after a second debridement in good condition. One month later she underwent reconstructive surgical treatment. Besides extensive FG and multiple comorbidity she was successfully managed with good outcome. Fournier's gangrene remains a life-threatening and fulminant disease which need urgent diagnosis and aggressive medical and surgical treatment, to achieve a reduction in long term complications and mortality rate.

\section{Introduction}

Fournier's gangrene, was first described by French venereologist Jean Alfred Fournier in 1883, as a fulminant type of gangrene in male patients with lesions in their genitals (Fournier, 1883). Fournier's gangrene is a life-threatening condition defined as necrotizing fasciitis of the genital, perianal and perineal regions which can rapidly extent to the lower extremities, abdominal wall or retroperitoneal space (Eke, 2000; Korkut et al., 2003). Fournier's gangrene a rare condition with male predominance and high mortality rate ( $73 \%)$ (Romero et al., 2016). The most common risk factors for development of Fournier's gangrene are: diabetes mellitus, alcoholism, malnutrition, smoking, malignancies, immunosuppressive therapy, obesity, liver or kidney failure (Sorensen and Krieger, 2016). Early diagnosis and adequate initial treatment including resuscitation, broad-spectrum antibiotics and aggressive surgical debridement are crucial to successful outcome.

\section{Case report}

A 59-years-old female patient with obesity and medical history of arterial hypertension and type 2 diabetes was hospitalized as an emergency case with diabetic ketoacidosis and sepsis. Anamnestic data showed an initial perianal skin lesion, fourteen days prior to hospitalization. The patient was treated with a local surgical incision in secondary-level hospital, and after the intervention, she was discharged at home with advice for further treatment with antibiotics. After one week, despite antibiotic treatment, the patient condition was getting worse dayto-day, so she underwent medical check-up in the same hospital, but due to the serious progression of a local lesion and bad medical condition, she was immediately referred to tertiary-level hospital. On physical examination patient presented an extensive gangrenous lesion located perianal, perineal, which spread through hypogastric region, gluteal and sacrococcygeal region. Physical examination showed blood pressure of $80 / 40 \mathrm{~mm} \mathrm{Hg}$, a heart rate of 100 beats per minute and a body temperature of $39.1^{\circ} \mathrm{C}$. Blood tests indicated a systematic inflammation response white blood cell $-19.5 \times 10^{9} / \mathrm{I}$ and C-reactive protein level of $422.8 \mathrm{mg} / \mathrm{dl}$. Random blood glucose level was $45.9 \mathrm{mmol} / \mathrm{I}$, so uncontrolled diabetes was diagnosed. In addition, laboratory tests showed high concentration of creatinine $-220 \mu \mathrm{mol} / \mathrm{I}$ and blood urea - $22.8 \mathrm{mmol} / \mathrm{l}$, signs of anemia - hemoglobin concentration - 


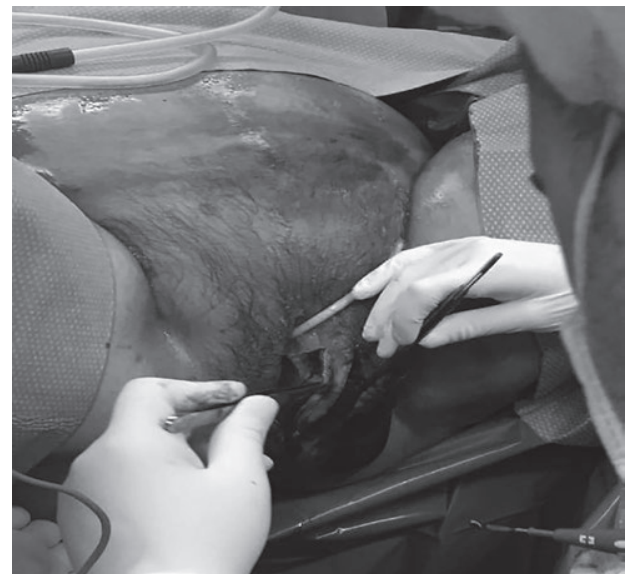

Figure 1 - Fournier's gangrene (necrotic lesions).

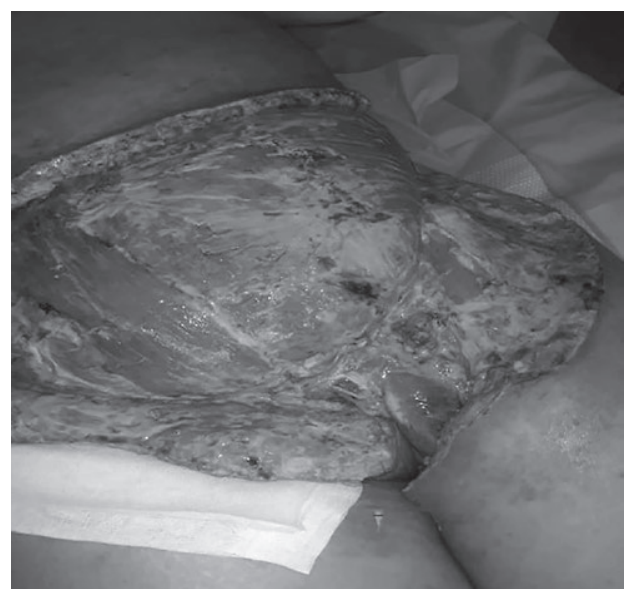

Figure $3-4^{\text {th }}$ postoperative day.

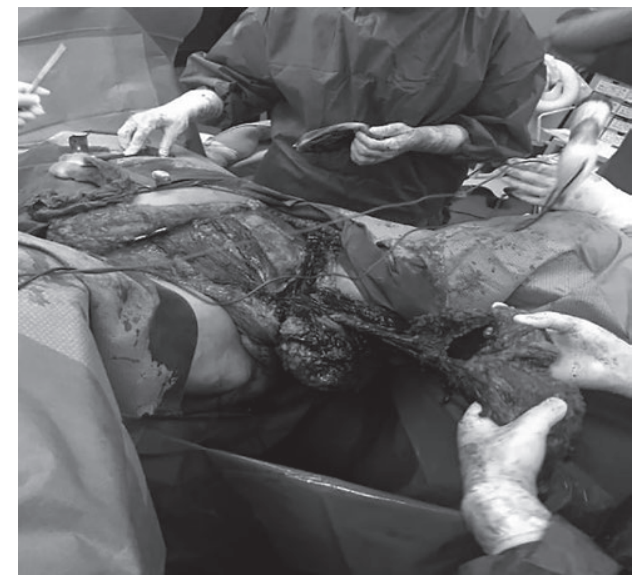

Figure 2 - Necrectomia. Debridement. Lavage. No II.

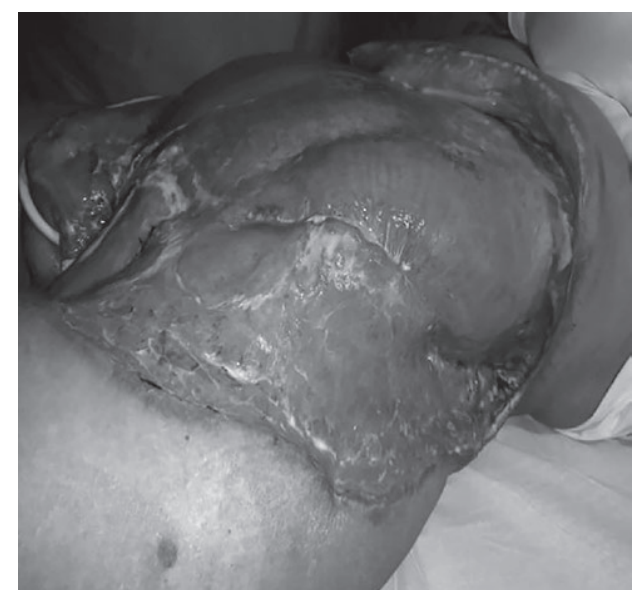

Figure 4 - Postsurgical wound after second debridement.

$100 \mathrm{~g} / \mathrm{l}$, red blood cells $-3.6 \times 10^{12} / \mathrm{l}$, hematocrit $-0.31 \%$. Based on the history, physical examination, laboratory analysis a diagnosis of sepsis and Fournier's gangrene was considered (Dg. Sepsis. Fasciitis necroticans regio perinealis, regio perianalis, labium majorum lat. sin, regio inguinalis billateralis, regio hypogastrici et umbilici, reg. lumbalis lat. sin, reg. femoris medialis lat. sin. et reg. sacralis.) (Figure 1). After initial resuscitation with intravenous fluids, broad-spectrum antibiotics (Imipenem/Cilastin $3 \mathrm{~g} /$ day, Vancomycin $2 \mathrm{~g} /$ day) and (IV) insulin infusion, the patient underwent emergency surgery under general anesthesia. An aggressive debridement of all necrotic areas down to viable tissue was made (Necrectomia. Debridement. Lavage. No II) (Figures 2 and 3). 


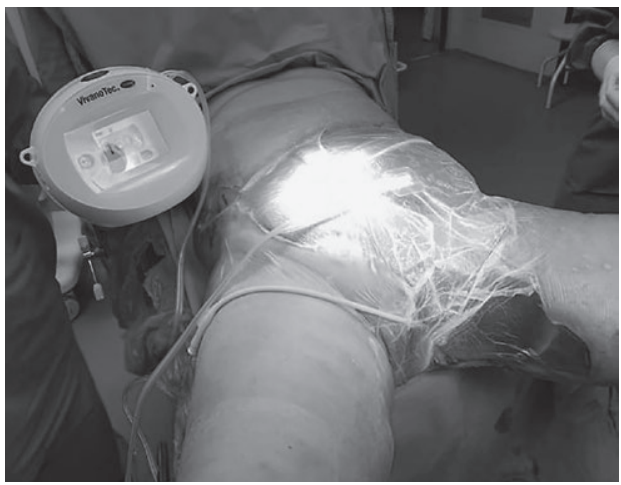

Figure 5 - Negative pressure wound therapy (NPWT).

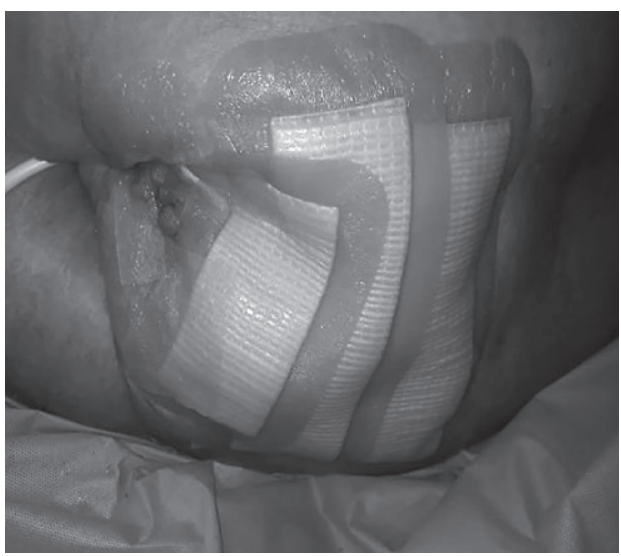

Figure 7 - Dressing the wound on gluteal and sacrococcygeal region with Granuflex and Hydro Clean.

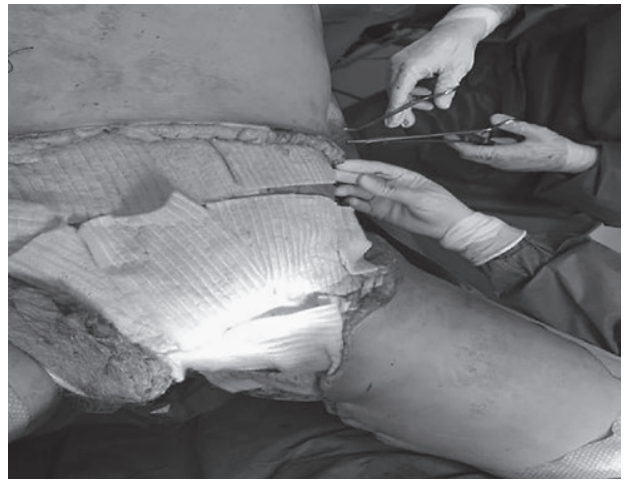

Figure 6 - Dressing the wound with Aquacell Ag.

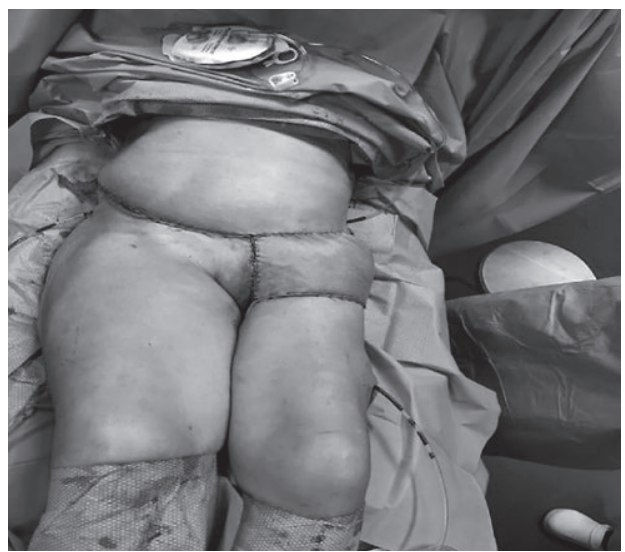

Figure 8 - Post reconstruction.

After first debridement the open wound was dressed with Aquacell Ag. Postoperative, on $4^{\text {th }}$ day, necrosis appeared again and second debridement was indicated (Figure 4).

After second debridement the wound was dressed with Hydro Clean, Granuflex and Aquacell $\mathrm{Ag}$ and negative pressure wound therapy was indicated. Postsurgical treatments of wound are shown on Figures 5-7.

Febrile status continued after second debridement, so wound cultures were collected and were identified: Pseudomonas aeruginosa, Proteus mirabilis, Enterococcus and Citrobacter freundii. After wound culture results, antibiotics were changed according to antibiogram: amp. Gentamycin $240 \mathrm{mg} /$ day and amp. Piperacillin/ Tazobactam $18 \mathrm{~g} /$ day. The febrile status continued and blood was collected for 
hemoculture where was identified Acinetobacter, that was the reason for new antibiotic approach, first with Colistin 3000,000 IE/day (10 days) and then amp. Meropenem IV $2 \mathrm{~g} /$ day and amp. Clindamycin 1,800 mg/day in next five days. During hospitalization besides antibiotics was administered anticoagulant and supportive therapy, glycemic control and parenteral nutrition.

Patient was discharged home five weeks after a second debridement in good condition with granulation of wound, with advice to continue with wound toilet at home, antibiotic therapy tab. Citeral $1 \mathrm{~g} /$ day, next 7 days and anticoagulant therapy amp. Clexane $40 \mathrm{mg}$.

After few weeks the patient returned at the University Clinic of Plastic and Reconstructive Surgery in Skopje for reconstructive surgery (Figure 8).

\section{Discussion}

Fournier's gangrene is a type of necrotizing fasciitis of the genital, perianal, and perineal regions. It is a serious surgical problem with high mortality rate (Hota, 2012). This condition is more common in men, but a literature data shows a high incidence of $31.6 \%$ in women (Czymek et al., 2010). About 60\% of patients with Fournier's gangrene have diabetes (Puvanendran et al., 2009). Pathophysiologically, Fournier's gangrene starts with microthrombosis of the small subcutaneous vessels which derives to skin gangrene, subsequent rapid spread of bacteria along the muscular fascia and relatively poor blood supply. The major clinical manifestation of Fournier's gangrene is: systemic inflammatory response syndrome, sepsis and extensive skin changes as a result of necrosis such as red/purple patches, erythema and swelling. Urgent diagnosis and treatment are crucial in the prognosis (Eke, 2000; Korkut et al., 2003). Diagnosis of Fournier's gangrene commonly is based on a clinical manifestation. Management of Fournier's gangrene basically depends on multidisciplinary approach. Initial resuscitation with fluid therapy and stabilization of cardiopulmonary function in patients with septic shock is very important at the time of presentation. In case of diabetic ketoacidosis is necessary immediately to start with intravenous insulin therapy. Aggressive surgical debridement of necrotized tissue in combination with broad-spectrum antibiotics is the main pathway in treatment of Fournier's gangrene. The removal of all the necrotized tissue is important to stop the spreading of the infection and subsequently elimination of systemic effects of toxins and bacteria (Czymek et al., 2009). Local wound care after surgical debridement is very important. Dressings the wound with Aqacell Ag, Granuflex, Hydro Clean and negative pressure wound therapy have been advocated. Negative pressure wound therapy have shown enhanced granulation tissue and reduction in wound surface area as a result of stimulation of fibroblasts activity, capillary dilatation, increasing circulation around the wound (Al Fadhli et al., 2009). With adequate surgical debridement, local wound care and antibiotic therapy, healthy granulation tissue appears. In case of significant tissue loss, especially in genitalia and perineum which leads to high morbidity, reconstructive procedure may be considered (Zaba et al., 2009). 


\section{Conclusion}

We can conclude that Fournier's gangrene still remains a diagnostic and therapeutic challenge, due to the fact that it is a rare medical condition with high mortality rate. Fournier's gangrene requires an urgent diagnosis and aggressive medical and surgical treatment, to achieve a reduction in long-term complications and mortality. We recommended adequate initial surgical therapy and multidisciplinary approach in treatment, for good patient outcome, especially in cases with multiple comorbidities.

\section{References}

Al Fadhli, A., Alexander, G., Kanjoor, J. R. (2009) Versatile use of vacuum-assisted healing in fifty patients. Indian J. Plast. Surg. 42, 161-168.

Czymek, R., Hildebrand, P., Kleemann, M., Roblick, U., Hoffmann, M., Jungbluth, C. T., Bruch, H. P. B., Kujath, P. (2009) New insights into the epidemiology and etiology of Fournier's gangrene: a review of 33 patients. Infection 37(4), 306-312.

Czymek, R., Frank, P., Limmer, S., Schmidt, A., Jungbluth, T., Roblick, U., Bürk, C., Bruch, H. P., Kujath, P. (2010) Fournier's gangrene: Is the female gender a risk factor? Langenbecks Arch. Surg. 395(2), 173-180.

Eke, N. (2000) Fournier's gangrene: a review of 1726 cases. Br. J. Surg. 87(6), 718-728.

Fournier, J. A. (1883) Gangrene foudroyante de la verge. Med. Prat. 4, 589-597.

Hota, P. K. (2012) Fournier's gangrene: report of 2 cases. Case Rep. Emerg. Med. 2012, 984195.

Korkut, M., Içöz, G., Dayangaç, M., Akgün, E., Yeniay, L., Erdoğan, O., Cal, C. (2003) Outcome analysis in patients with Fournier's gangrene: report of 45 cases. Dis. Colon Rectum 46, 649-652.

Puvanendran, R., Huey, J. C., Pasupathy, S. (2009) Necrotizing fasciitis. Can. Fam. Physician 55, 981-987.

Romero, C. R., Gracida, M. N., Romero, F. R., Romero, F. R. (2016) A case report of a woman with Fournier's gangrene and morbid obesity. Rev. Latinoam. Patol. Clin. Med. Lab. 63(2), 82-86.

Sorensen, M. D., Krieger, J. N. (2016) Fournier's gangrene: Epidemiology and outcomes in the general US population. Urol. Int. 97, 249-259.

Zaba, R., Grzybowski, A., Prokop, J., Zaba, Z., Zaba, C. (2009) Fournier's gangrene: Historical survey, current status, and case description. Med. Sci. Monit. 15(2), CS34-CS39. 